\title{
An Energy-Efficient User-Centric Approach for High-Capacity 5G Heterogeneous Cellular Networks
}

\author{
Abdulziz M. Ghaleb, Ali Mohammed Mansoor and Rodina Ahmad \\ Dep. of Software Engineering. Faculty of Computer Science \& IT, University of Malaya, Malaysia
}

\begin{abstract}
Today's cellular networks (3G/4G) do not scale well in heterogeneous networks (HetNets) of multiple technologies that employ network-centric (NC) model. This destabilization is due to the need for coordination and management of multiple layers of the HetNets that the NC models cannot provide. User-centric (UC) approach is one of the key enablers of 5G wireless cellular networks for rapid recovering from network failures and ensuring certain communication capability for the users. In this paper, we present resource-aware energy-saving technique based on the UC model for LTE-A HetNets. We formulate an optimization problem for UC as a mixed linear integer programming (MILP) that minimizes the total power consumption (Energy Efficiency) while respecting the data rate per user and propose a low complexity iterative algorithm to user terminal (UE)-eNodeB association. In UC model, UE possessing terminal intelligence can establish the transmission and reception with different cells within the LTEA HetNet assuming the existence of coordination between the different cells in the network. The performance is evaluated in terms of energy saving in the uplink and downlink and the added capacity to the network (data rate). The evaluation is carried out by comparing a UC model against a NC model with the same simulation setup. The results show significant percentage of energy saving at eNodeBs and UEs in a UC model. Also, system capacity is enhanced in the UC model in both the uplink and downlink due to utilizing best channel gain for transmission and reception.
\end{abstract}

Keywords-Energy efficiency; HetNets; green networks; usercentric; network-centric; $5 G$

\section{INTRODUCTION}

Today's $3 \mathrm{G}$ and $4 \mathrm{G}$ cellular networks are principally designed based on cell-centric or network-centric (NC) model with a focus on peak rate and spectral efficiency improvements. In the $5 \mathrm{G}$ era, dense deployment of heterogeneous network (HetNet) architecture will shift towards user-centric (UC) model to deliver a uniform connectivity experience. Therefore, 5G networks will require advanced source coding and advanced radio access networks. The objective is to significantly improve the flexibility of deployment and connectivity by making them more and more user-oriented [1]. The relationship between the downlink and the uplink in HetNets is different from that of the homogeneous ones. The transmit power of all transmitters in the uplink is roughly the same (independent of distance and amount of traffic) since all UEs are running off batteries. In contrast, there exist transmit power disparities between different eNodeB (eNB) types in the downlink (up to $20 \mathrm{~dB}$ ) [2].

The efficient deployment of HetNets in $5 \mathrm{G}$ era calls for new disruptive technologies in a way that allows the corresponding information to flow in multiple data streams through different sets of heterogeneous nodes [4]. 5G networks should achieve combined gains in three categories: extreme densification and offloading, increased bandwidth and increased spectral efficiency in order to support 1,000-fold gains in capacity and connections for at least 100 billion devices. The demand in capacity gain would increase the consumed energy by the network by a factor of 100 [1]-[5].

Therefore, the NC architecture should evolve into a UC one, and uplink and downlink could be considered as two separate networks. Each network will require different models for interference, cell association, and throughput [2]-[4]. In UC architecture, the UE has a crucial role in establishing the connectivity with the eNBs. The UE can decide whether to establish connectivity with the same cell or with different cells in the uplink and downlink communication. In this perspective, new carrier type was proposed in [5] where user/data and control planes can be separated in UEs by small cells at higher frequency bands (mmWave). This is expected to reduce the frequent handover between small cells and macrocell and among small cells. Hence, the connectivity can be maintained even when using small cells and higher frequency bands since connectivity and mobility is provided by the control plane [6].

\section{A. Motivation for this Work}

The current works does not involve any performance evaluation of the UC model in term of power efficiency, capacity improvement or Quality of Service (QoS). The motivation for this work is to provide good insights of the performance of UC model deployment in future 5G networks. In this work, we have formulated an optimization problem for $\mathrm{UC}$ as mixed linear integer programming (MILP) that minimizes the total power consumption while respecting the data rate per user and proposed a low complexity iterative algorithm to UEeNB association. The paper provides an evaluation for the UC model in LTE-A HetNets in terms of energy saving at both eNBs and UEs and the added capacity to the network. Two sets of simulation experiments for different number of UEs (reflecting the network load) were carried out with the same setup; one for NC model and one for UC model. The collected results show the percentage of energy saving in the UC model compared to the NC model and additional gained data rate (data rate in the $\mathrm{NC}$ model subtracted from data rate in the UC model). The results show significant energy saving (up to $15 \%$ in the downlink and $6 \%$ in the uplink) and capacity enhancement in UC model. It is noteworthy that some claims that NC strategies are better than UC ones in terms of achievable throughput but are worse in terms of computational complexity in certain scenarios (i.e., LTE/WiFi coexistence) [7]. This opens research door for further investigation of the UC model as well as hybrid or joint user and network architecture. 
The rest of the paper is arranged as follows. Section II provides an overview of the related works. Section III provides a detailed description of the UC-based network model. The problem is formulated in Section IV and proposed method described in Section V. The results are presented in Section VI. Finally Section VII concludes the paper.

\section{RELATED WORKS}

Some works have considered the UC architecture for wired networks or Internet for self-organizing, autonomic networks. The architecture is used for sharing network services and resources by installing the device as the owner and controller of its personal data [8]-[11]. Recently, some works have envisioned the UC architecture as one of the core features of the $5 \mathrm{G}$ networks [1], [2], [4]-[6], [8], [12]-[14]. Not all the authors considered the full UC paradigm; some of them either considered the separation of uplink and downlink [2] or separating control and data planes [5].

To the best of the authors' knowledge, little work has been done on the evaluation of the UC architecture for wireless communication. The authors of [15] studied the dynamic user association decoupled UL-DL time division duplexing (TDD)-based networks to balance the UL and DL loads in different small cells. The authors of [16] presented a transmission/reception scheme for LTE/LTE-A HetNets that exploits the concept of Coordinated Multipoint (CoMP). The authors of [17] we presented device-centric design, implementation, and testing of optimized data aggregation mechanisms for file downloading and video streaming applications. The uplink and downlink transmissions of a UE are established with different cells assuming the existence of coordination between the cells. In this scheme, the UE is associated to a small cell for uplink transmission and to macrocell for downlink reception. The authors in [18] investigated the potential to enable emergency communications with different radio access technologies such as LTE and WLAN which are the candidates for direct communication in emergency cases [19]. However, the main focus was to enable better emergency communication. It was not in the context of $5 \mathrm{G}$ networks, and there was no considerations for using this feature for network efficiency and self-organization. The most significant work done in this regard is in [20] where the authors studied the decoupling of downlink and uplink based on simulation of LTE field trial network in a dense urban HetNet deployment. The authors considered downlink cell association based on the received power and uplink cell association based on the pathloss.

\section{Network MOdel}

We consider a a LTE-A HetNet deployed in a given geographical area divided into equal-size cells where an eNB is placed at the center of each cell. The area also includes smaller cells (micro, femto, pico) placed either within the macrocells or to bridge the coverage gaps. ENBs are classified as macro, micro, pico and femto eNBs based on both their transmit power and their antenna heights. In LTE air interface, Orthogonal Frequency Division Multiple Access (OFDMA) is used for the downlink access mechanism and the Single Carrier - Frequency Division Multiple Access (SC-FDMA) is used for the uplink. For OFDM-based access schemes, the available spectrum is divided into subcarriers in the frequency domain. In LTE, the spectrum is divided into resource blocks (RBs). Each RB is constituted by 12 consecutive subcarriers for a fixed duration of $1 \mathrm{~ms}$. In the UC model, the uplink and downlink are decoupled and are considered two separate networks. The deployment scenario is shown in Fig. 1.

\section{A. Energy Consumption Model}

1) Power Consumption Model for eNodeBs: For simplicity, we consider that each eNB in the macro and small cells are equipped with an omni-directional antenna. The $j$ th active eNB consumed power $P_{j}^{\mathrm{eNB}}$ is computed as follows [21]:

$$
P_{j}^{\mathrm{eNB}}=a_{j} P_{j}^{\mathrm{tx}}+b_{j},
$$

where $P_{j}^{\text {tx }}$ denotes the radiated power of the $j$ th eNB. The coefficient $a_{j}$ corresponds to the radiated power consumed due to feeder and amplifier losses. The term $b_{j}$ is the fixed power offset which is consumed by the site independently of the transmitted power and depends on the eNB type.

2) Power Consumption Model for UEs: Each UE in the network is considered to be equipped with a set of omnidirectional antennas $\mathcal{N}_{i}^{\text {ant }}$ and can communicate with macro and small cells (open access for femto cells). Assuming that the $i$ th $\mathrm{UE}$ is connected to a set of eNBs $\mathcal{N}_{\mathrm{eNB}}^{\mathrm{DL}, i}$ in the downlink and to a set of eNBs $\mathcal{N}_{\mathrm{eNB}}^{\mathrm{UL}, i}$ in the uplink according to the suggested UC model for 5G [1], [2], [4], [6], [16], [22] and given that the $i$ th user is connected to the $j$ th eNB through set of antennas, then the consumed power $P_{i}^{\mathrm{UE}}$ of the $i$ th running mobile is computed as follows:

$$
P_{i}^{\mathrm{UE}}=m_{i}^{l} \sum_{l \in \mathcal{N}_{i}^{a n t}} \sum_{j \in \mathcal{N}_{\mathrm{eNB}}^{\mathrm{UL}, i}} P_{i, j}^{\mathrm{tx}, l}+n_{i},
$$

where $P_{i, j}^{\mathrm{tx}, l}$ corresponds to the radiated power of the $l$ th antenna of the $i$ th UE connected to the $j$ th eNB. The coefficient $m_{i}^{l}$ corresponds to the radiated power consumed due to system losses which varies form one antenna to another and $n_{i}$ is the fixed power consumed to keep the mobile on.

The eNB's energy consumption is segregated into two types namely, the static energy consumption and the dynamic energy consumption. When turned on, each eNB consumes a constant amount of energy (fixed power) depending on its type regardless of the traffic load. This amount of energy is always required just for the equipment to be powered on. Similarly, UE's energy consumption is divided into static and dynamic energy consumption. The second part is the adaptive power consumption which is proportional to the transmission density. For the UEs, they are assumed to be on all the time and there is nothing to optimize regarding their static power consumption. Henceforward, this paper focuses on optimizing both saving the eNBs static and dynamic energy consumption as well as the UEs adaptive energy consumption. The overall power consumed by the HetNet infrastructure and UEs $E_{H e t}$ for a $T$ hours of time can be represented by the sum of energy consumed by all active eNBs and UEs as follows:

$$
E_{\mathrm{Het}}=\left(\sum_{j=1}^{N_{\mathrm{eNB}}} P_{j}^{\mathrm{eNB}}+\sum_{i=1}^{N_{\mathrm{UEs}}} P_{i, j}^{\mathrm{UE}}\right) \times \frac{T}{1000}(\mathrm{kWh}),
$$




\section{Cell 1}

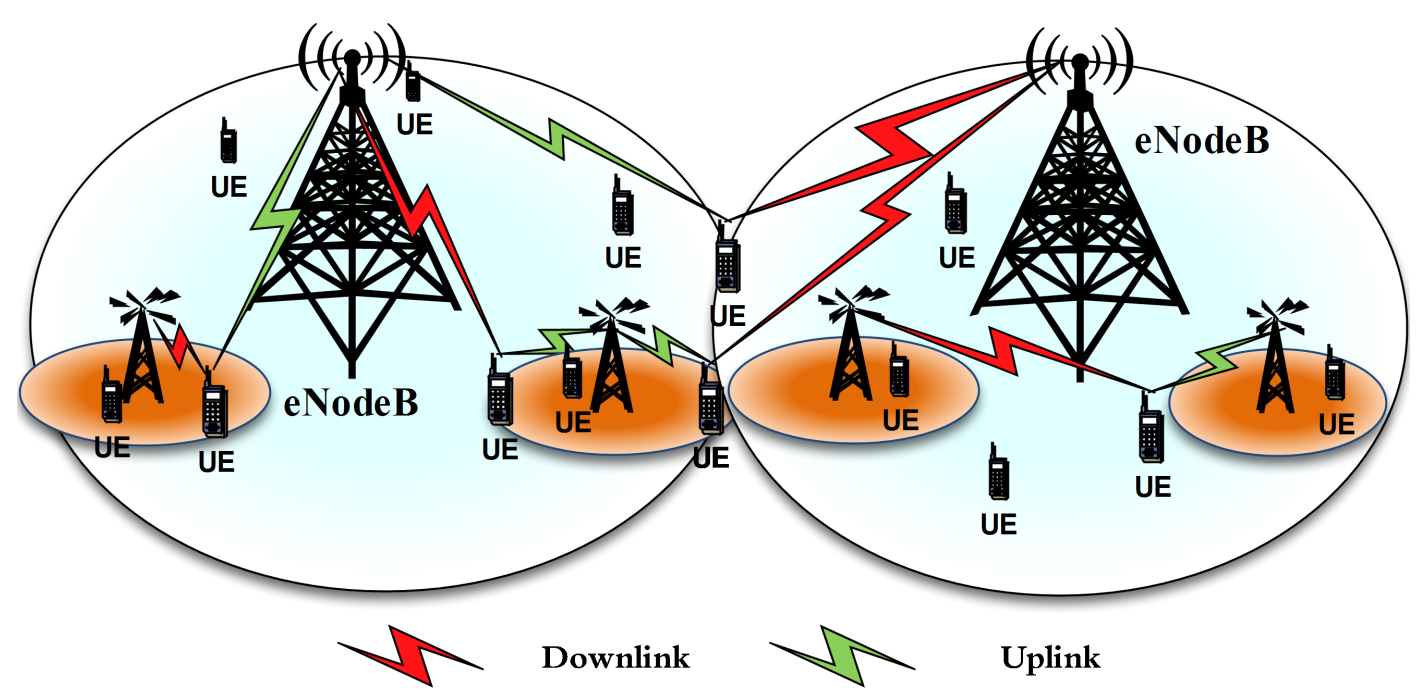

Fig. 1. Deployment scenario with UC model.

\section{B. Channel Model}

The channel gain for both uplink and downlink over subcarrier $s$ between $i$ th UE and $j$ th eNB is given by [23]:

$$
H_{i, s, j, \mathrm{~dB}}=\left(-\kappa-v \log _{10} d_{i, j}\right)-\xi_{i, s, j}+10 \log _{10} F_{i, s, j}
$$

where the first term represents the propagation loss with $\kappa$ being the path loss constant, $d_{i, j}$ being the distance in $\mathrm{km}$ from the $i$ th $\mathrm{UE}$ to the $j$ the eNB and $v$ being the path loss exponent. The second term, $\xi_{i, s, j}$, represents the zero-mean log-normal shadowing with a standard deviation $\sigma_{\xi}$, while $F_{i, s, j}$ corresponds to Rayleigh fading. The notation $H_{i, s, j}^{\mathrm{UL}}$ and $H_{i, s, j}^{\mathrm{DL}}$ will be used to differentiate between uplink and downlink channel gains, respectively. The LTE bandwidth is defined as a function of the number of RBs multiplied by the RB bandwidth, $B=N_{\mathrm{RB}} \times B_{\mathrm{RB}}(\mathrm{kHz})$. It can be expressed in terms of number of subcarriers and subcarrier bandwidth as $B=N_{\text {sub }} \times B_{\text {sub }}(\mathrm{kHz})$.

\section{Data Rates Calculation}

1) Data Rates in the Downlink: Letting $s_{i, j}$ be a subcarrier assigned by the $j$ th eNB to the $i$ th $\mathrm{UE}, \mathcal{I}_{s, i, j}^{\mathrm{DL}}$ be the set of downlink subcarriers allocated to the $i$ th UE from the $j$ th eNB and $R_{i}^{\mathrm{DL}}$ the achievable dwonlink rate of the $i$ th UE. The set of subcarriers given to the $i$ th UE by the HetNet in the downlink is denoted as $\mathcal{I}_{s, i}^{\mathrm{DL}}$. The OFDMA data rate of $i$ th UE supported by the $j$ th eNB is given by:

$$
R_{i, j}^{\mathrm{DL}}\left(P_{j, \text { max }}^{\mathrm{tx}}, \mathcal{I}_{s, i, j}^{\mathrm{DL}}\right)=\sum_{s \in \mathcal{I}_{s, i, j}^{\mathrm{DL}}} B_{s} \cdot \log _{2}\left(1+\gamma_{i, s, j}^{\mathrm{DL}}\right)
$$

where $\gamma_{i, s, j}^{\mathrm{DL}}$ is the downlink SINR of the $i$ th UE over subcarrier $s$ transmitted form the $j$ th eNB and is given by:

$$
\gamma_{i, s, j}^{\mathrm{DL}}=\frac{P_{s, j}^{\mathrm{tx}} H_{i, s, j}^{\mathrm{DL}}}{I_{s, i, j}^{\mathrm{DL}}+\sigma_{s, i, j}^{2}}
$$

where $H_{i, s, j}^{\mathrm{DL}}$ is the channel gain of the $i$ th $\mathrm{UE}$ over subcarrier $s, \sigma_{s, i, j}^{2}$ is the noise power over subcarrier $s$ in the receiver of the $i$ th $\mathrm{UE}$, and $I_{s, i, j}^{\mathrm{DL}}$ is the interference on subcarrier $s$ measured at the receiver of the $i$ th UE. The total data rate provided to the $i$ th UE by the network is given by:

$$
R_{i}^{\mathrm{DL}}=\sum_{j \in \mathcal{N}_{\mathrm{eNB}}^{i}} R_{i, j}^{\mathrm{DL}}
$$

The total data rate provided to the $i$ th UE by the network should be equal to or greater than a threshold value, $R_{i, t h}^{\mathrm{DL}}$, in order to provide the QoS requested by the user based on the contract. Letting $\mathcal{N}_{\mathrm{UE}}^{j}$ set of UEs attached to the $j$ th eNB, the total data rate that cell $j$ can support is given by:

$$
R_{j}^{\mathrm{DL}}=\sum_{j \in \mathcal{N}_{\mathrm{UE}}^{j}} R_{i, j}^{\mathrm{DL}}
$$

We assume that bandwidth varies from one eNB to another, so does the total number of subcarriers $N_{\text {sub }}^{\text {DL }}$ for cell $j$ in the downlink. As we seek to come out with optimized realistic solutions for power allocation, we consider non-uniform or adaptive power transmission over the subcarriers, i.e., $P_{s, j}^{\mathrm{tx}}$ is not constant. This allows eNBs to adjust their transmit power levels according to the distance of the UE, interference and modulation and coding scheme (MCS).

2) Data Rates in the Uplink: According to UC model, UE can be associated with one or more eNB in the uplink. Letting $\mathcal{I}_{s, i, j}^{\mathrm{UL}}$ be the set of uplink subcarriers granted to the $i$ th $\mathrm{UE}$ from $j$ th eNB, $P_{i, j}^{\mathrm{UE}}$ the total transmit power of the $i$ th $\mathrm{UE}$ and $R_{i, j}^{\mathrm{UL}}$ its achievable rate in the uplink, the set of subcarriers guaranteed to the $i$ th $\mathrm{UE}$ by the HetNet in the uplink $\mathcal{I}_{s, i}^{\mathrm{UL}}$ then, the SC-FDMA data rate of the $i$ th UE is given by:

$$
\begin{aligned}
R_{i, j}^{\mathrm{UL}}\left(P_{i, j}^{\mathrm{UE}}, \mathcal{I}_{s, i, j}^{\mathrm{UL}}\right)= & B_{\mathrm{sub}}\left|\mathcal{I}_{s, i, j}^{\mathrm{UL}}\right| \cdot \\
& \log _{2}\left(1+\gamma_{i, j}^{\mathrm{UL}}\left(P_{i, j}^{\mathrm{UE}}, \mathcal{I}_{s, i, j}^{\mathrm{UL}}\right)\right)
\end{aligned}
$$


where $\left|\mathcal{I}_{s, i, j}^{\mathrm{UL}}\right|$ is the cardinality of $\mathcal{I}_{s, i, j}^{\mathrm{UL}}$ and $\gamma_{i, j}^{\mathrm{UL}}\left(P_{i, j}^{\mathrm{UE}}, \mathcal{I}_{s, i, j}^{\mathrm{UL}}\right)$ is the SINR of the $i$ th UE after frequency domain equalization at the receiver. The uplink SINR of the $i$ th UE over subcarrier $s$ served by $j$ th eNB and is given by [24]:

$$
\gamma_{i, s, j}^{\mathrm{UL}}=\frac{P_{i, s, j}^{\mathrm{UE}} H_{i, s, j}^{\mathrm{UL}}}{I_{s, j}^{\mathrm{UL}}+\sigma_{s, j}^{2}}
$$

where $H_{i, s, j}^{\mathrm{UL}}$ is the channel gain between the $i$ th $\mathrm{UE}$ and the $j$ th eNB over subcarrier $s, \sigma_{s, j}^{2}$ is the noise power over subcarrier $s$ at the $j$ th eNB, $P_{i, s, j}^{\mathrm{UL}}$ is the power transmitted by the $i$ th UE over subcarrier $s$ in the $j$ th cell.

\section{PROBLEM FORMULATION}

In both uplink and downlink, amount of data rate depends on both number of assigned subcarriers and SINR. SINR is a function of the transmit power and the link quality. However, increasing the power is not necessarily a good choice since it leads, of course, to higher power consumption and increase the interference which degrades the link quality specially in ultra dense deployment of $5 \mathrm{G}$ systems. UC approach can reduce the interference and ensure energy savings in designing green wireless cellular networks with higher capacity. With the decoupling of uplink and downlink, UE can be associated with different eNBs in the uplink and downlink so that data rate is maximized with minimum power consumption. Assuming that UE has full knowledge of the channel status which can be sensed or collected from the eNB, the UE will choose the best link for downlink and uplink data transmission. The total power consumption over all subcarriers has to be less or equal to the maximum transmission power of the eNB denoted by $P_{j \text {,max }}^{\mathrm{tx}}$. The LTE standard mandates that the RBs allocated to a single user in the uplink be consecutive with equal power allocation over their subcarriers [25], [26]. The model be formulated as follows:

\section{- Parameters:}

$\mathcal{N}_{\text {eNB }}$ : set of the deployed eNBs within the HetNet $\mathcal{N}_{\mathrm{UE}}$ : set of subscribers in the area

- Decision Variables:

$\delta_{i, j}^{\mathrm{DL}}=\left\{\begin{array}{l}1 \text { if the } i \text { th UE is associated to the } j \text { th eNB } \\ \text { in the downlink, } \\ 0 \text { otherwise. }\end{array}\right.$ $\eta_{i, j}^{\mathrm{UL}}=\left\{\begin{array}{l}1 \text { if the } i \text { th UE is associated to the } j \text { th eNB } \\ \text { in the uplink, } \\ 0 \text { otherwise. }\end{array}\right.$ $\vartheta_{x, i, j}^{\mathrm{DL}}=\left\{\begin{array}{c}1 \text { if subcarriers } s_{x} \text { is allocated to the } i \text { th UE } \\ \text { from the } j \text { th eNB in the downlink, } \\ 0 \text { otherwise. }\end{array}\right.$ $\epsilon_{x, i, j}^{\mathrm{UL}}=\left\{\begin{array}{c}1 \text { if subcarriers } s_{x} \text { is allocated to the } i \text { th UE } \\ \text { from the } j \text { th eNB in the uplink, } \\ 0 \text { otherwise. }\end{array}\right.$ $\psi_{i}^{\mathrm{DL}}=\left\{\begin{array}{l}1 \text { if } R_{i}^{\mathrm{DL}} \geq R_{i, t h}^{\mathrm{DL}} \\ 0 \text { otherwise. }\end{array}\right.$ $\varrho_{i}^{\mathrm{DL}}=\left\{\begin{array}{l}1 \text { if } R_{i}^{\mathrm{UL}} \geq R_{i, t h}^{\mathrm{UL}} \\ 0 \text { otherwise. }\end{array}\right.$

\section{- Mathematical Model:}

$$
\begin{aligned}
\text { Minimize: } & \sum_{\text {Subject to: }} P_{j}^{\mathrm{eNB}}+\sum_{i=1}^{N_{\mathrm{UEs}}} P_{i, j}^{\mathrm{UE}} P_{s, j}^{\mathrm{tx}} \leq P_{j, \max }^{\mathrm{tx}} \\
& \left|\mathcal{I}_{s, i, j}^{\mathrm{UL}}\right| \times P_{i, s}^{\mathrm{UL}} \leq P_{i, \max }^{\mathrm{tx}} \\
& R_{i}^{\mathrm{DL}} \geq R_{i, t h}^{\mathrm{DL}} \\
& R_{i}^{\mathrm{UL}} \geq R_{i, t h}^{\mathrm{UL}} \\
& 1 \leq \sum \delta_{i, j}^{\mathrm{DL}} \leq\left|\mathcal{N}_{\mathrm{eNB}}\right| \\
& 1 \leq \sum_{i, j}^{\mathrm{UL}} \leq\left|\mathcal{N}_{\mathrm{eNB}}\right| \\
& \sum_{i \in\left\{1,\left|\mathcal{N}_{\mathrm{UE}}\right|\right\}} \vartheta_{i, j}^{\mathrm{DL}} \leq 1 \\
& \sum \epsilon_{i, j}^{\mathrm{UL}} \leq 1 \forall \mathrm{UE} \mathrm{E}_{i} \in \mathcal{N}_{\mathrm{UE}} \& \forall \mathrm{eNB} \mathrm{N}_{i} \in \mathcal{N}_{\mathrm{eNB}} \\
& \sum \vartheta_{i}^{\mathrm{DL}}=1 \cdot x: x \in\left\{1,\left|\mathcal{I}^{\mathrm{DL}}\right|\right\} \\
& \sum \epsilon_{i}^{\mathrm{UL}}=2 \cdot y: y \in\left\{1,\left|\mathcal{I}^{\mathrm{UL}}\right| / 2\right\}
\end{aligned}
$$

where (12) and (13) ensure that eNB and UE do not exceed the maximum allowed transmit power while (14) and (15) ensure that the data rates are equal or greater than the required threshold values in order to respect the communication QoS for the downlink and uplink, respectively. Cell association in the uplink an downlink is ensured by (16) and (16), respectively.

\section{PRoposed SCHEME}

The UE is assumed to have some terminal intelligence and ability to establish connectivity with different cells within the LTE-A HetNet assuming the existence of coordination between the different cells in the network. The UE decides which cells to choose for uplink and downlink transmissions such that the energy efficiency is maximized. Algorithm 1 illustrates the implementation at the UE.

First, the UE searches the available eNBs, $\mathcal{N}_{\mathrm{eNB}}^{i}$, that can establish communication with. Next, the UE calculates the channel gain in the uplink and downlink based on the interference followed by the power consumption required to transmit with the required data rate. If the power required does not exceed a certain limit, the eNB is added to the uplink and/or downlink eNB candidates pool, $\mathcal{N}_{\mathrm{eNB}}^{\mathrm{DL}, i}$ and/or $\mathcal{N}_{\text {eNB }}^{\mathrm{UL}, i}$. The set of candidate eNBs for the uplink and downlink communications are sorted according to the required power for the uplink and downlink transmission. The UE secures resources from $\mathcal{N}_{\mathrm{eNB}}^{\mathrm{DL}, i}$ and $\mathcal{N}_{\mathrm{eNB}}^{\mathrm{UL}, i}$ for the uplink and downlink communications starting with the eNB requiring less power till satisfying the required data rate. The rest of the eNBs are then neglected. The same approach can be applied for NC with only one difference, which is the eNB of the uplink will be the one of the downlink.

\section{NumERICAL RESUlTS}

This section presents and analyzes the simulation results and outlines energy-saving and capacity improvement of the 


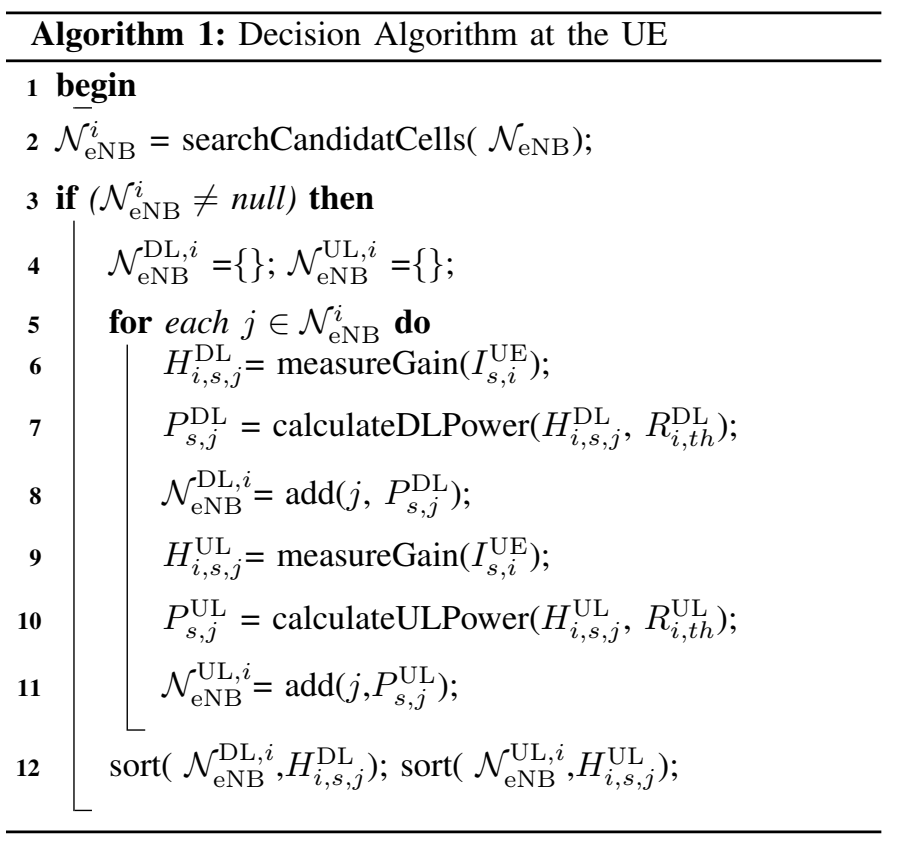

LTE-A networks with dense 5G deployments. MATLAB simulation results obtained by comparing the performance of a UC model and NC model. We consider a 2-by-2 $\mathrm{km}$ area with four LTE-A macro cells of radius $500 \mathrm{~m}$ and 12 small cells of radius $125 \mathrm{~m}$. Each macro eNB is placed at the cell center and surrounded by three small eNBs, all eNBs are equipped with omnidirectional antennas. The number of users are varied between 50 and 400 UEs which indicates the load variation. Table I summarizes the default simulation parameters settings.

We evaluate the performance of the UC architecture in term of energy saving and added capacity to the network which ultimately indicate the impact on the QoS. The ES (\%) is percentage of the reduction of consumed energy by the system when deploying the UC model to the energy consumed with $\mathrm{NC}$ model deployment and is measured as $\mathrm{ES}(\%)=\frac{E_{\mathrm{NC}}-E_{\mathrm{UC}}}{E_{\mathrm{NC}}}$, where $E_{\mathrm{NC}}$ is the energy consumed with $\mathrm{NC}$ deployment and $E_{\mathrm{UC}}$ is the energy consumed with UC deployment. The added capacity indicates the difference between the data rate of the system with UC and NC models.

In 3GPP LTE, channel quality indication values describe a range of targeted MCSs. The overall size of the Transport Block and the number of allocated $\mathrm{RBs}$ are given as the effective spectral efficiency. UEs can be associated with one eNB in the downlink and one eNB in the uplink. The UC model brings many attractive advantages. Fig. 2 shows the number of users that are associated with different eNBs in the uplink and downlink according to the UC model.

With UC, one or more eNBs can be utilized the downlink transmission and the uplink transmission from any other eNBs. This enables the UE to handle the asymmetric traffic. Since UE can exchange data in either downlink or uplink utilizing the best portion of spectrum with best channel gain, it can transmit same amount of data with less energy consumption and/or increase system capacity by using higher-order MCS. Fig. 3 and 4 show the energy saving at the eNBs and UEs,
TABLE I. DEFAult Parameters

\begin{tabular}{|l|c|}
\hline Parameter & Settings \\
\hline Area & 2-by-2 km \\
\hline No. of eNBs & 16 \\
\hline Bearer Type & Default \\
\hline Path loss Model & Free space \\
\hline Transmission Mode & SISO \\
\hline Frequency Reuse & 1 \\
\hline Cyclic Prefix & Normal \\
\hline Duplexing Mode & FDD \\
\hline DL Bandwidth & $60(3 \times 20) \mathrm{MHz}$ \\
\hline UL Bandwidth & $40(2 \times 20) \mathrm{MHz}$ \\
\hline BLER & $10^{-4}$ \\
\hline eNB Antenna Type & Omnidirectional \\
\hline UE Antenna Type & Omnidirectional \\
\hline macro eNB $P_{j, \text { max }}^{\text {tx }}$ & 40 Watt/46dBm \\
\hline small eNB $P_{j, \text { max }}^{\text {tx }}$ & 20 Watt/43dBm \\
\hline UE $P_{i, \text { max }}^{\text {tx }}$ & 125 mWatt/21dBm \\
\hline$R_{i, t h}^{\text {DL } h}$ & $5 \mathrm{Mbps}$ \\
\hline$R_{i, t h}^{\text {LL }}$ & $2 \mathrm{Mbps}$ \\
\hline
\end{tabular}

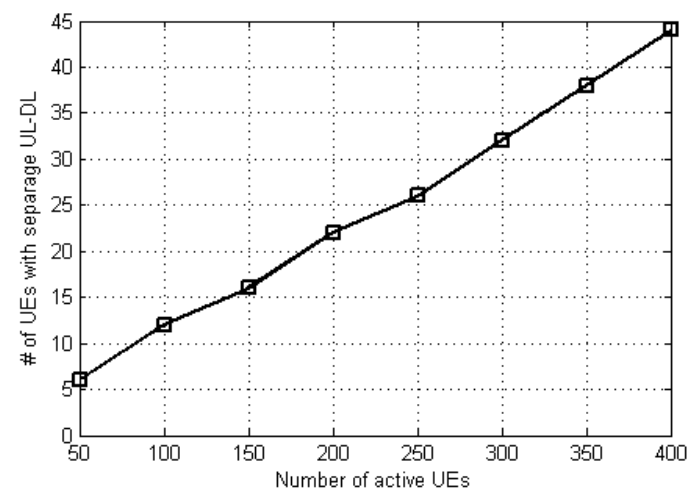

Fig. 2. Number of UEs with separate uplink-downlink connections.

respectively. With 150 active UEs and less, on-off techniques could be implemented to the eNBs which optimize the energy saving, up to $15 \%$. Some eNBs were switched off when active users are 50 and 100 UEs while only one eNB could be switched off with 150 active UEs.

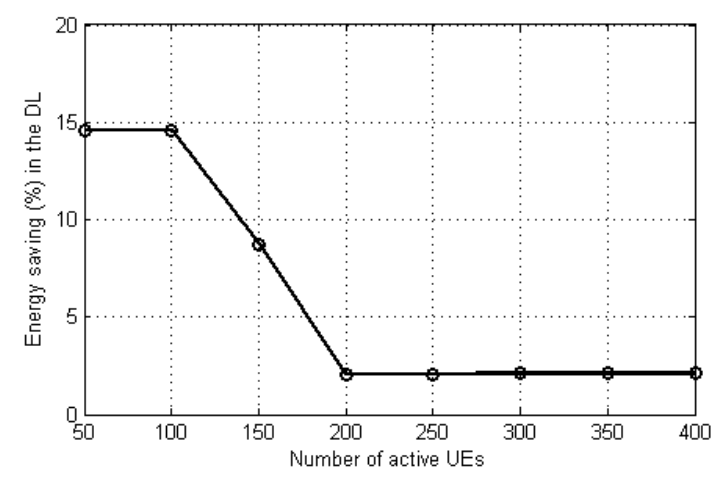

Fig. 3. Energy saving (\%) in the downlink (at eNBs). 
With implemented UC model, the energy saving at UE's transmit energy is about $5 \%$ of the total energy consumed without implementing UC model. Saving energy at the UEs prolong the battery life and has good impact on human health. The data rate in the downlink is generally higher than that of the uplink in LTE, according to [27]. Here, data rate is affected, in both directions, by the MCS order and number of users. Again, better link quality will significantly increase the data rate. The total data rate provided by the network is in factor of Gbps.

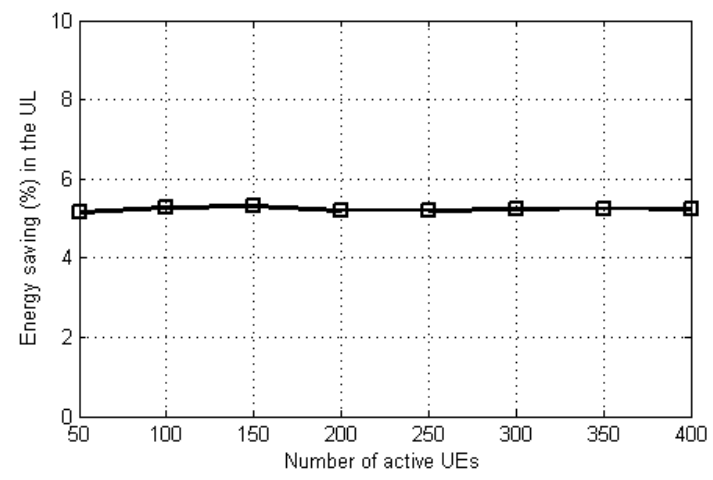

Fig. 4. Energy saving (\%) in the Uplink (at UEs).

Fig. 5 shows the added data rate in the uplink and downlink, the added capacity due to the decoupling of the uplink and downlink. The results show that the added data rate is proportional to the number of active UEs and number of UEs with separate uplink/downlink connections. The added capacity is obtained because the number of users served with UC (less UE outage) is higher than that when networkcentric model is implemented since UC offers more degree of freedom due to uplink-downlink separation (ability to connect to different multiple eNobeBs) and offer better link quality.

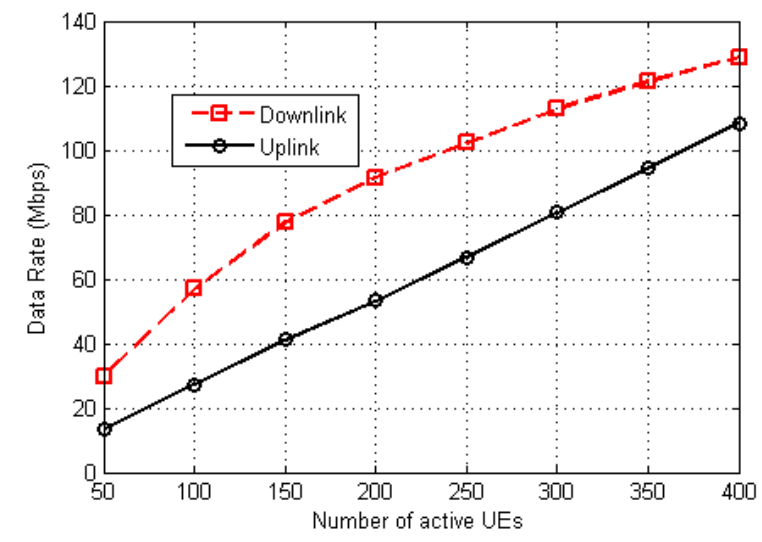

Fig. 5. Added capacity (data rate) to the network.

Table II summarizes the obtained results for the different number of active UEs. With increasing the number of UEs and considering fluctuating radio resources where channel gain is fluctuating, UC model is expected to add more efficiency to the network in terms of added data rates and energy savings.
TABLE II. SUMMARY OF OBTAINED RESULTS

\begin{tabular}{|l|l|l|l|l|l|}
\hline $\begin{array}{l}\text { Active } \\
\text { UEs }\end{array}$ & $\begin{array}{l}\text { UL/DL } \\
\text { UEs }\end{array}$ & $\begin{array}{l}\text { DL ES } \\
(\%)\end{array}$ & $\begin{array}{l}\text { UL ES } \\
(\%)\end{array}$ & $\begin{array}{l}\text { DL Data } \\
\text { Rate } \\
(\mathbf{M b p s})\end{array}$ & $\begin{array}{l}\text { UL Data } \\
\text { Rate } \\
(\text { Mbps })\end{array}$ \\
\hline 50 & 6 & 14.6 & 5.15 & 29.70 & 13.39 \\
\hline 100 & 12 & 14.6 & 5.26 & 56.94 & 27.24 \\
\hline 150 & 16 & 8.73 & 5.30 & 77.55 & 41.09 \\
\hline 200 & 22 & 2.07 & 5.18 & 91.35 & 53.18 \\
\hline 250 & 26 & 2.07 & 5.18 & 102.29 & 66.73 \\
\hline 300 & 32 & 2.09 & 5.22 & 112.82 & 80.59 \\
\hline 350 & 38 & 2.1 & 5.25 & 121.10 & 94.33 \\
\hline 400 & 44 & 2.12 & 5.23 & 128.84 & 108.27 \\
\hline
\end{tabular}

\section{CONCLUSIONS}

$5 \mathrm{G}$ radio access technologies aims to increase the data rates of UEs while reducing the energy consumption per amount of data. User-centric model is foreseen as an interesting feature for minimizing the power consumption at the UEs and eNBs as well. It enables transmission with better link quality and/or, possibly, transmission to the nearest eNB for at least one direction (uplink or downlink) which requires less power for the same amount of data. The results show significant amount of energy savings at the UEs and eNBs. With cooperation between the uplink and downlink, user-centric model adds a degree of freedom to the network planning where a UEs of specific cell can be associated with other cells in uplink and downlink and their cell can be switched off to save energy. Future work include modeling a comprehensive framework for energy efficiency in 5G network including the disruptive $5 \mathrm{G}$ features such as massive MIMO. These features can be included to add a degree of freedom to the advanced self organizing $5 \mathrm{G}$ network for energy efficiency. The investigation of hybrid/joint user and network centric is also very interesting area of research.

\section{ACKNOWLEDGMENT}

This study is supported by the Fundamental Research Grant Scheme (FRGS), Project: FP007-2016 from Ministry of Higher Education, Malaysia.

\section{REFERENCES}

[1] B. Bangerter, S. Talwar, R. Arefi, and K. Stewart, "Networks and devices for the 5g era," IEEE Communications Magazine, vol. 52, no. 2, pp. 90-96, February 2014.

[2] J. Andrews, "Seven ways that hetnets are a cellular paradigm shift," IEEE Communications Magazine, vol. 51, no. 3, pp. 136-144, March 2013.

[3] B. Finley and A. Basaure, "Benefits of Mobile End User Network Switching and Multihoming," CoRR, vol. 1705.01398, 2017.

[4] F. Boccardi, R. Heath, A. Lozano, T. Marzetta, and P. Popovski, "Five disruptive technology directions for 5G," IEEE Communications Magazine, vol. 52, no. 2, pp. 74-80, February 2014.

[5] Y. Kishiyama, A. Benjebbour, T. Nakamura, and H. Ishii, "Future steps of LTE-A: evolution toward integration of local area and wide area systems," IEEE Wireless Communications, vol. 20, no. 1, pp. 12-18, February 2013.

[6] W. H. Chin, Z. Fan, and R. J. Haines, "Emerging technologies and research challenges for 5G wireless networks," IEEE Wireless Communications, vol. 21, no. 12, pp. 106-112, April 2014.

[7] G. Dandachi, S. E. Elayoubi, T. Chahed and N. Chendeb, "NetworkCentric Versus User-Centric Multihoming Strategies in LTE/WiFi Networks," in IEEE Transactions on Vehicular Technology, vol. 66, no. 5, pp. 4188-4199, May 2017. 
[8] J. Yelmo, J. del Alamo, R. Trapero, P. Falcarin, J. Yi, B. Cairo, and C. Baladron', "A user-centric service creation approach for next generation networks," in Innovations in NGN: Future Network and Services, First ITU-T Kaleidoscope Academic Conference, May 2008, pp. 211-218.

[9] R. Sofia, P. Mendes, and J. Moreira, Waldir, "User-centric networking: Living-examples and challenges ahead," in User-Centric Networking, ser. Lecture Notes in Social Networks, A. Aldini and A. Bogliolo, Eds. Springer International Publishing, 2014, pp. 25-51.

[10] R. Sofia, "User-centric networking: Bringing the home network to the core," in User-Centric Networking, ser. Lecture Notes in Social Networks, A. Aldini and A. Bogliolo, Eds. Springer International Publishing, 2014, pp. 3-23.

[11] I. Baumgart and F. Hartmann, "Towards secure user-centric networking: Service-oriented and decentralized social networks," in Fifth IEEE Conference on Self-Adaptive and Self-Organizing Systems Workshops, Ann Arbor, MI, Oct 2011, pp. 3-8.

[12] X. Xing, T. Jing, W. Zhou, X. Cheng, Y. Huo, and H. Liu, "Routing in user-centric networks," IEEE Communications Magazine, vol. 52, no. 9, pp. 44-51, September 2014.

[13] M. Katsarakis, G. Fortetsanakis, P. Charonyktakis, A. Kostopoulos, and M. Papadopouli, "On user-centric tools for qoe-based recommendation and real-time analysis of large-scale markets," IEEE Communications Magazine, vol. 52, no. 9, pp. 37-43, September 2014.

[14] S. ping Yeh, S. Talwar, G. Wu, N. Himayat, and K. Johnsson, "Capacity and coverage enhancement in heterogeneous networks," IEEE Wireless Communications, vol. 18, no. 3, pp. 32-38, June 2011.

[15] M. S. ElBamby, M. Bennis and M. Latva-aho, "UL/DL decoupled user association in dynamic TDD small cell networks," in in International Symposium on Wireless Communication Systems, Brussels, 2015, pp. 456-460.

[16] A. M. Ghaleb, E. Yaacoub, and D. Chieng, "Physically separated uplink and downlink transmissions in LTE HetNets based on CoMP concepts," in IET International Conference on Frontiers of Communications, Networks and Applications, November 2014, pp. 1-7.

[17] S. Sharafeddine, K. Jahed, and M. Fawaz, "Optimized device centric aggregation mechanisms for mobile devices with multiple wireless interfaces," in Computer Networks, Volume 129, Part 1, 2017, Pages 1-16, ISSN 1389-1286.

[18] Y. Gao, Y. Li, H. Yu, X. Wang, and S. Gao, "Performance analysis of the separation of uplink and downlink under lte-advanced system level simulation: An energy aware point of view," in 2nd International Conference on Computer Science and Network Technology, Dec 2012, pp. 1289-1293.

[19] M. Macuha, D. Amgalan, and T. Derham, "Device-centric approach to improve resiliency of emergency communication," in IEEE Region 10 Humanitarian Technology Conference, Aug 2013, pp. 124-129.

[20] H. Elshaer, F. Boccardi, M. Dohler, and R. Irmer, "Downlink and uplink decoupling: a disruptive architectural design for 5g networks," IEEE Global Communications Conference, Aug 2014, pp. 1798-1803.

[21] F. Richter, A. Fehske, and G. Fettweis, "Energy efficiency aspects of base station deployment strategies for cellular networks," in Proc. of the 70th IEEE Vehicular Technology Conference Fall, Anchorage, Alaska, USA, Sep. 2009.

[22] C.-X. Wang, F. Haider, X. Gao, X.-H. You, Y. Yang, D. Yuan, H. Aggoune, H. Haas, S. Fletcher, and E. Hepsaydir, "Cellular architecture and key technologies for $5 \mathrm{~g}$ wireless communication networks," IEEE Communications Magazine, vol. 52, no. 2, pp. 122-130, February 2014.

[23] A. Goldsmith, Wireless Communications. Cambridge University Press, 2005.

[24] J. Lim, H.G. Myung, K. Oh, and D.J. Goodman, "Channel-dependent scheduling of uplink single carrier FDMA systems," in Proc. of the 64th IEEE Vehicular Technology Conference Fall (VTC 2006-Fall), Montreal, Quebec, Canada, Sep. 2006.

[25] H. G. Myung and D. J. Goodman, Single Carrier FDMA: A New Air Interface for Long Term Evolution. Wiley, 2008.

[26] 3rd Generation Partnership Project (3GPP), "3GPP TS 36.211 3GPP TSG RAN Evolved Universal Terrestrial Radio Access (E-UTRA) Physical Channels and Modulation, version 8.3.0, Release 8," 3GPP, Tech. Rep., 2008.

[27] A. Ghaleb, D. Chieng, A. Ting, A. Abdulkafi, K.-C. Lim, and H.-S. Lim, "Throughput performance insights of LTE release 8: Malaysia's perspective," in 9th International Wireless Communications and Mobile Computing Conference, July 2013, pp. 258-263. 